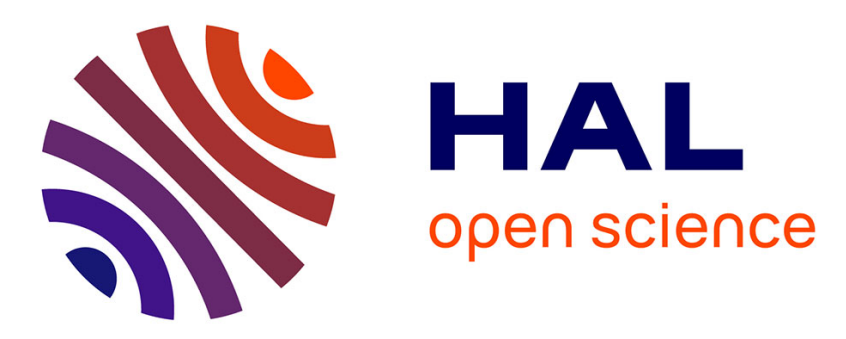

\title{
Periodic solution finder for an impact oscillator with a drift
}

\author{
Ekaterina Pavlovskaia, Marian Wiercigroch
}

\section{To cite this version:}

Ekaterina Pavlovskaia, Marian Wiercigroch. Periodic solution finder for an impact oscillator with a drift. Journal of Sound and Vibration, 2003, 267, pp.893 - 911. 10.1016/S0022-460X(03)00193-7 . hal-01510819

\section{HAL Id: hal-01510819 \\ https://hal.science/hal-01510819}

Submitted on 20 Apr 2017

HAL is a multi-disciplinary open access archive for the deposit and dissemination of scientific research documents, whether they are published or not. The documents may come from teaching and research institutions in France or abroad, or from public or private research centers.
L'archive ouverte pluridisciplinaire HAL, est destinée au dépôt et à la diffusion de documents scientifiques de niveau recherche, publiés ou non, émanant des établissements d'enseignement et de recherche français ou étrangers, des laboratoires publics ou privés. 


\title{
Periodic solution finder for an impact oscillator with a drift
}

\author{
E. Pavlovskaia, M. Wiercigroch \\ Centre for Applied Dynamics Research, Department of Engineering, King's College, Fraser Noble Building, Aberdeen \\ University, Aberdeen AB24 3UE, UK
}

In this paper, an efficient semi-analytical method is developed to compute periodic solutions for a new model of an imp ad oscillator with a drift, which exp lans the progression mechanism in vibro-impact systems and can be used to op timize their $\mathrm{p}$ erfomance. The method constructs a $\mathrm{p}$ eriodic response assuming that each period is comprised of a sequence of distinct phases for which analytical solutions are known. For example, a period may consist of the following sequential phases: (I) contact with progression, (II) contact without $\mathrm{p}$ rogresion, (III) no contact and (IV) contact without $\mathrm{p}$ rogresion. Using this information, a system of four piecewise linear first order differential equations is transformed to a system of non-linear algebraic equations. The method allows one to accurately predict a range of control parameters for which the best progression rates are obtained.

\section{Introduction}

Impacting systems have numerous engineering applications, for example, pile driving [1], percussive drilling [2,3] and ground moling [4]. For a better illustration of the problem, consider a particular engineering application such as vibro-impact ground moling. During operation, the generated impact forces are used to drive a penetrating head into the ground. This can result in complex dynamic responses, as the system not only oscillates but also moves forward. A combination of oscillatory and progressive motion has been recently studied by Pavlovskaia et al. $[5,6]$ using an elasto-viscous model of the soil, which is more realistic than previously reported (see, for example, Refs. [7,8]). From those studies and others including experimental ones (e.g., $[3,4])$, it is clear that the best progression rates are achieved when the system responses are 
periodic. Strictly speaking, when the impacting mass oscillates with a period one motion and steadily moves forward.

Non-linear dynamics of impacting oscillators has received a considerable theoretical and experimental attention in the past (see for example Refs. [9-16]). Practical aspects of vibro-impact and percussion systems have been vigorously studied for many years. For example, works by Kobrinskii [17] and Babitsky [18] provide detailed analyses assuming that the dynamics of vibroimpact systems can be reduced to only oscillatory motion. This might be justifiable for some regimes of operation, but in general, the progression has an influence on the oscillatory motion, and therefore a comprehensive model accounting for both motions is required. This has been pointed out in the recent paper by Pavlovskaia et al. [5]; however, the method to analyze the oscillatory progressive motion has only been mentioned. Therefore, the main aim of this paper is to present a robust algorithm capable of determining periodic solutions (responses) for an impact oscillator which is also drifting from its initial position.

\section{Governing equations}

A simple dynamical system shown in Fig. 1 is considered, where a mass $m$ is excited by an external force containing a harmonic component of amplitude $P_{d}$, frequency $\Omega$ and phase shift $\varphi$, and a static component, $P_{s}$. The slider has weightless top and bottom plates connected to each other by a linear elasto-viscous pair of stiffness $k$ and damping $c$. Similar to the stick-slip phenomena reported for example in Refs. [19,20], the progressive motion of the mass occurs when the force acting on the slider exceeds the threshold of the dry friction force $P_{f}$. As proposed in Ref. [5] $X_{m}, X_{t}, X_{b}$ represent the absolute displacements of the mass, slider top and slider bottom, respectively. It is assumed that the model operates in a horizontal plane, or the gravitational force is compensated. At the initial moment, there is a distance separating the mass and the slider top called a gap, $E$. Now introduce a new variable $U=X_{t}-X_{m}+E$ to monitor this distance.

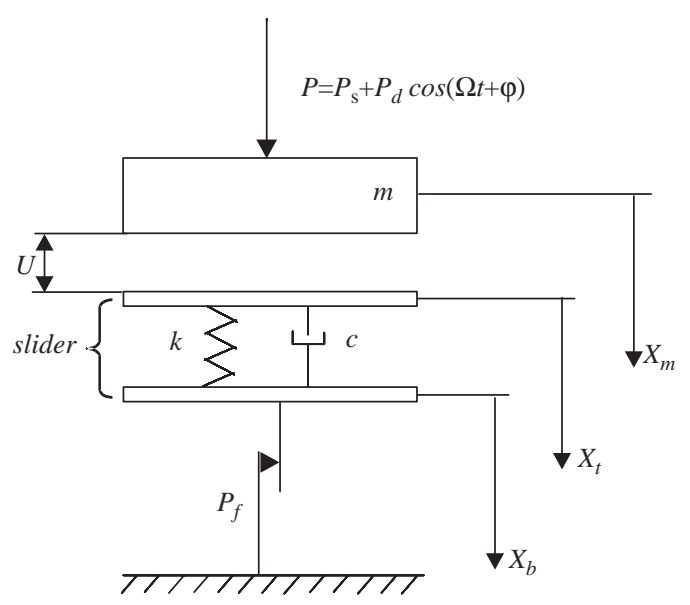

Fig. 1. Physical model of an impact oscillator with a drift. 
For the case when the mass and the slider move separately, the dynamics of the system is described by one second and two first order differential equations:

$$
\begin{gathered}
m \ddot{X}_{m}=P_{s}+P_{d} \cos (\Omega t+\varphi), \\
c\left(\dot{X}_{t}-\dot{X}_{b}\right)+k\left(X_{t}-X_{b}\right)=0, \\
\dot{X}_{b}=0 .
\end{gathered}
$$

When the mass and the slider are in contact, their motion is described by one second order and one first order differential equation, which can be either oscillatory

$$
\begin{gathered}
m \ddot{X}_{m}+c\left(\dot{X}_{t}-\dot{X}_{b}\right)+k\left(X_{t}-X_{b}\right)=P_{s}+P_{d} \cos (\Omega t+\varphi), \\
\dot{X}_{b}=0,
\end{gathered}
$$

or progressive

$$
\begin{gathered}
m \ddot{X}_{m}=-P_{f}+P_{s}+P_{d} \cos (\Omega t+\varphi), \\
c\left(\dot{X}_{t}-\dot{X}_{b}\right)+k\left(X_{t}-X_{b}\right)=P_{f} .
\end{gathered}
$$

Note that for Eqs. (2) and (3), the distance between the mass and the slider top $U=0$, which means that the displacement of the slider top, $X_{t}$ is in phase with the displacement of the mass, $X_{m}$, but is smaller by a gap, $X_{t}=X_{m}-E$.

The equations of motion (1)-(3) were transformed to sets of first order differential equations using the following non-dimensional variables [5]:

$$
\begin{gathered}
\tau=\Omega_{0} t, \quad x=\frac{k}{P_{\max }} X_{m}, \quad y=\frac{\mathrm{d} x}{\mathrm{~d} \tau}=\frac{k}{\Omega_{0} P_{\max }} \dot{X}_{m}, \\
z=\frac{k}{P_{\max }} X_{t}, \quad v=\frac{k}{P_{\max }} X_{b}, \quad u=\frac{k}{P_{\max }} U
\end{gathered}
$$

and parameters

$$
\begin{aligned}
& \omega=\frac{\Omega}{\Omega_{0}}, \quad \Omega_{0}=\sqrt{\frac{k}{m}}, \quad a=\frac{P_{d}}{P_{\max }}, \quad b=\frac{P_{s}}{P_{\max }}, \quad d=\frac{P_{f}}{P_{\max }}, \\
& \xi=\frac{c}{2 m \Omega_{0}}, \quad e=\frac{k}{P_{\max }} E,
\end{aligned}
$$

where $P_{\max }$ is the normalized threshold force.

As has been discussed above, the considered system can operate in one of the following modes: no contact, contact without the progression of the slider, or contact with the progression of the slider. For each of these modes a careful consideration will be given next. For simplicity of further analysis, the dimensionless friction threshold force, $d$ is set to $1, e=0.02$ and $\varphi=\pi / 2$.

\subsection{No contact}

If the distance between the mass and the slider top is greater than zero, $u>0$, then the mass and the slider top move separately. The motion of the mass can be determined from the following set 
of equations:

$$
\begin{gathered}
x^{\prime}=y, \\
y^{\prime}=a \cos (\omega \tau+\varphi)+b,
\end{gathered}
$$

where ' denotes $\mathrm{d} / \mathrm{d} \tau$. Velocities at the top and the bottom of the slider are

$$
\begin{gathered}
z^{\prime}=-\frac{1}{2 \xi}(z-v), \\
v^{\prime}=0 .
\end{gathered}
$$

\subsection{Contact without progression}

This mode occurs when the distance between the mass and the slider top is equal to zero, $u=0$, and the force acting on the mass from the slider is greater than zero but smaller than the threshold of the dry friction force. This can be expressed as

$$
0<2 \xi z^{\prime}+(z-v)<1 .
$$

The mass and the slider top move together without progression, and the second equation of (4) gains additional elastic and viscous terms:

$$
\begin{gathered}
x^{\prime}=y, \\
y^{\prime}=-2 \xi z^{\prime}-(z-v)+a \cos (\omega \tau+\varphi)+b .
\end{gathered}
$$

The velocity of the slider top is equal to the velocity of the mass, and the displacement of the slider top is in phase with the mass displacement but differs by gap, $e$ :

$$
z^{\prime}=x^{\prime}, \quad x=z+e .
$$

When there is no progression, the bottom of the slider remains stationary, hence its velocity is equal to zero, $v^{\prime}=0$.

\subsection{Contact with progression}

When the distance between the mass and the slider top is equal to zero, $u \leqslant 0$, and the force acting on the mass is greater than the threshold of dry friction force which can be described as

$$
2 \xi z^{\prime}+(z-v) \geqslant 1,
$$

then the mass and the top and the bottom of the slider move together, and progression takes place. Equations of motion for the mass are

$$
\begin{gathered}
x^{\prime}=y, \\
y^{\prime}=a \cos (\omega \tau+\varphi)+b-1 .
\end{gathered}
$$


The displacement and the velocity of the slider top are as before (see Eq. (9)). The velocity of the slider bottom motion can be calculated from the expression

$$
v^{\prime}=z^{\prime}+\frac{1}{2 \xi}(z-v-1) .
$$

\section{Algorithm for determining periodic solutions}

Let us now focus on periodic regimes as they are most beneficial from the practical point of view [7]. It should be noted that since the considered system is piecewise linear, its dynamic response can be constructed by stitching linear solutions at points of discontinuities. The following approach has been adopted. Initially, it is assumed that the displacement and velocity of the mass have certain (yet unknown) values. Starting from these values, the system is in one of the phases described in Section 2, and its analytical solution can be constructed. This enables one to calculate a time interval for which a particular phase of motion persists. The final displacements and velocities of the preceding phase define the initial conditions for the next phase. Finally, the initial conditions of the first phase are determined from the periodicity conditions. In Fig. 2, a typical sequence of period one motion is presented. It is comprised of

- Phase I-progression; the mass and the slider are in contact.

- Phase II - contact without progression; the mass and the slider are in contact but the slider bottom is not moving.

- Phase III-no contact; the mass and the slider are moving separately.

- Phase IV - contact without progression; the same as Phase II.

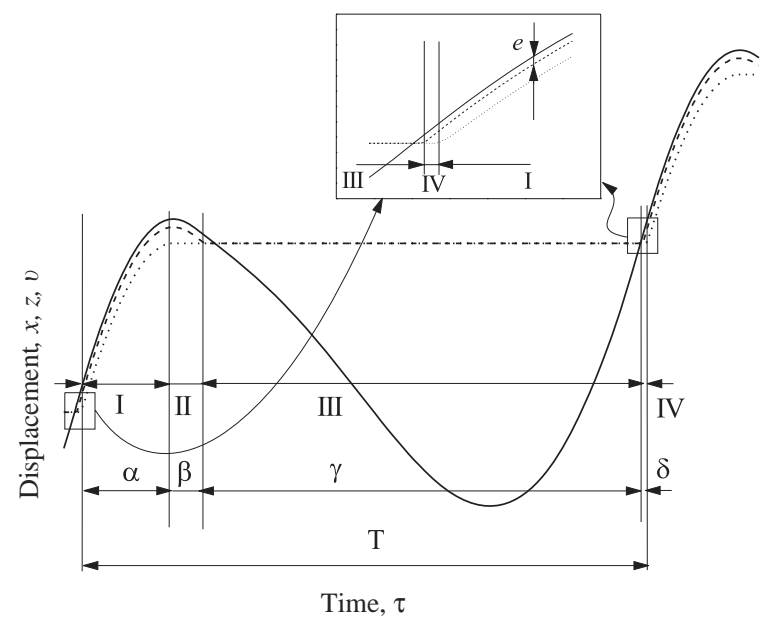

Fig. 2. One period broken into four phases of progression (I), contact without progression (II), no contact (III) and contact without progression (IV). Durations of these phases are $\alpha, \beta, \gamma$ and $\delta$, respectively. Solid, dashed and dotted lines mark displacements of the mass, the slider top and the slider bottom, respectively. 
The beginning of progression was chosen as an initial point. At this moment, the mass and the slider top are joined together, and the force acting on the mass from the slider has reached the threshold value. Since the velocities of the mass and the slider top are equal, the following relation between the initial displacement and velocity can be written as

$$
2 \xi y_{0}+\left(x_{0}-e-v_{0}\right)=1 .
$$

As the initial displacement of the slider bottom, $v_{0}$ does not influence the mass motion, it is set to zero. Then the following holds:

$$
x_{0}=1+e-2 \xi y_{0} .
$$

The other unknown is the time at this initial moment $\tau_{0}$. This means, that the phase shift between the external force and the system response at the beginning of the process needs to be determined:

$$
\psi_{0}=\varphi+\omega \tau_{0} .
$$

There are two periodic conditions for the mass displacement and velocity, which can be used:

$$
\begin{gathered}
x(\tau+T)=x(\tau)+\Delta, \\
y(\tau+T)=y(\tau),
\end{gathered}
$$

where $T$ is the period and $\Delta$ is progression of the slider per period. In the present study, the interest is in the periodic regime, where the period $T$ is equal to the period of external loading, $T=2 \pi / \omega$. Thus, three unknown functions $\psi_{0}, x_{0}$ and $y_{0}$ can be found from this condition together with Eq. (16). However, an arbitrary solution of these equations cannot guarantee that $x_{0}$ and $y_{0}$ will also satisfy Eq. (14). For that reason first a substitution of $x_{0}$ with the function of $y_{0}$ (expression (14)) is made, and then a special function $F$ to monitor the difference between the exact periodic solution and the one calculated for these arbitrary initial conditions is constructed:

$$
F=\sqrt{(x(\tau+T)-x(\tau)-\Delta)^{2}+(y(\tau+T)-y(\tau))^{2}} .
$$

If the minimum of this function is equal to zero, then the periodic regime exists, and the durations of all four stages can be determined. As this scheme is generic, the algorithm developed for calculating periodic solutions will be explained in detail.

\subsection{Phase I: progression}

The first phase is progression, and the consideration is started at the moment when this phase has just begun. Using the initial values of the unknown functions $\psi_{0}$ and $y_{0}$, a solution of Eq. (11) can be constructed as

$$
\begin{gathered}
x(\tau)=-\frac{a}{\omega^{2}}\left[\cos \left(\omega \tau+\psi_{0}\right)-\cos \left(\psi_{0}\right)\right]+\frac{1}{2}(b-1) \tau^{2}+y_{0} \tau-\frac{a \tau}{\omega} \sin \left(\psi_{0}\right)+x_{0}, \\
y(\tau)=\frac{a}{\omega}\left[\sin \left(\omega \tau+\psi_{0}\right)-\sin \left(\psi_{0}\right)\right]+(b-1) \tau+y_{0} .
\end{gathered}
$$


Having calculated $x(\tau)$ and assuming that $z(\tau)=x(\tau)-e$, the displacement of the slider bottom is found by integrating Eq. (12):

$$
v(\tau)=x(\tau)-e-1+2 \xi y_{0} \exp \left(-\frac{\tau}{2 \xi}\right) .
$$

When the progression condition (10) fails, a new phase, the contact without progression begins, and thus the end of the first phase can be determined from the following equation:

$$
y(\alpha)-y_{0} \exp \left(-\frac{\alpha}{2 \xi}\right)=0 .
$$

By substituting Eq. (19) into (21) the following expression is obtained:

$$
\frac{a}{\omega}\left[\sin \left(\omega \alpha+\psi_{0}\right)-\sin \left(\psi_{0}\right)\right]+(b-1) \alpha+y_{0}-y_{0} \exp \left(-\frac{\alpha}{2 \xi}\right)=0 .
$$

This enables one to calculate the duration of the first phase, $\alpha$, which is a function of the initial conditions, $y_{0}$ and $\psi_{0}$ :

$$
\alpha=\alpha\left(y_{0}, \psi_{0}\right) .
$$

Consequently, the progression of the slider per period, $\Delta$ can be expressed with respect to $\alpha$ as

$$
\Delta=v(\alpha)=x(\alpha)-e-1+2 \xi y_{0} \exp \left(-\frac{\alpha}{2 \xi}\right) .
$$

At this point, the progression phase has finished and a new phase, the contact without progression, has just begun. Hence, the initial conditions for the second phase are calculated from Eqs. (18) and (19) and

$$
x_{I}=x(\alpha), \quad y_{I}=y(\alpha)
$$

\subsection{Phase II: contact without progression}

In the second phase, the mass and the slider are moving together, but the force between them, which is acting on the slider is too small for the slider bottom to move. The motion of the mass is governed by Eq. (8), and the solution takes a form

$$
\begin{aligned}
x(\tilde{\tau})= & \exp (-\xi \tilde{\tau})\left[\mathscr{K}_{1} \cos \left(\tilde{\tau} \sqrt{1-\xi^{2}}\right)+\mathscr{K}_{2} \frac{\sin \left(\tilde{\tau} \sqrt{1-\xi^{2}}\right)}{\sqrt{1-\xi^{2}}}\right] \\
& +k_{1} \cos \left(\omega \tilde{\tau}+\psi_{1}\right)+k_{2} \sin \left(\omega \tilde{\tau}+\psi_{1}\right)+b+e+\Delta, \\
y(\tilde{\tau})= & \exp (-\xi \tilde{\tau})\left[\mathscr{K}_{3} \cos \left(\tilde{\tau} \sqrt{1-\xi^{2}}\right)+\mathscr{K}_{4} \frac{\sin \left(\tilde{\tau} \sqrt{1-\xi^{2}}\right)}{\sqrt{1-\xi^{2}}}\right] \\
& +\omega k_{2} \cos \left(\omega \tilde{\tau}+\psi_{1}\right)-\omega k_{1} \sin \left(\omega \tilde{\tau}+\psi_{1}\right),
\end{aligned}
$$


where

$$
\begin{aligned}
& \tilde{\tau}=\tau-\alpha, \\
& \psi_{1}=\psi_{0}+\omega \alpha, \\
& k_{1}=\frac{a\left(1-\omega^{2}\right)}{\left(1-\omega^{2}\right)^{2}+4 \xi^{2} \omega^{2}}, \quad k_{2}=\frac{2 a \xi \omega}{\left(1-\omega^{2}\right)^{2}+4 \xi^{2} \omega^{2}}, \\
& \mathscr{K}_{1}=x_{I}-k_{1} \cos \left(\psi_{1}\right)-k_{2} \sin \left(\psi_{1}\right)-b-e-\Delta, \\
& \mathscr{K}_{2}=y_{I}+\xi x_{I}-\xi b-\xi e-\xi \Delta+\left(-k_{1} \xi-k_{2} \omega\right) \cos \left(\psi_{1}\right)+\left(k_{1} \omega-k_{2} \xi\right) \sin \left(\psi_{1}\right), \\
& \mathscr{K}_{3}=y_{I}+\omega k_{1} \sin \left(\psi_{1}\right)-\omega k_{2} \sin \left(\psi_{1}\right), \\
& \mathscr{K}_{4}=-x_{I}+b+e+\Delta-\xi y_{I}+\left(k_{1}+k_{2} \omega \xi\right) \cos \left(\psi_{1}\right)+\left(-k_{1} \omega \xi+k_{2}\right) \sin \left(\psi_{1}\right) .
\end{aligned}
$$

$x_{I}$ and $y_{I}$ are determined from Eq. (25). The second phase ends when the force acting on the mass is equal to zero, so

$$
2 \xi y(\beta)+x(\beta)-e-\Delta=0,
$$

where $x$ and $y$ are calculated from Eq. (26). A functional relation for the interval $\beta$ is obtained by substituting Eqs. (24) and (26) into Eq. (27). This leads to a non-linear algebraic equation with unknown $y_{0}$ and $\psi_{0}$, which allows one to calculate the length of the second phase $\beta$ :

$$
\beta=\beta\left(y_{0}, \psi_{0}\right) \text {. }
$$

When $\beta$ is found, the initial conditions for the next phase can be calculated from Eq. (26) as

$$
x_{I I}=x(\beta), \quad y_{I I}=y(\beta), \quad z_{I I}=x(\beta)-e .
$$

In Fig. 3, trajectories of the mass (solid line) and the slider bottom (dashed line) during the first two phases for different values of the initial phase, $\psi_{0}$ are shown. It can be observed by looking at

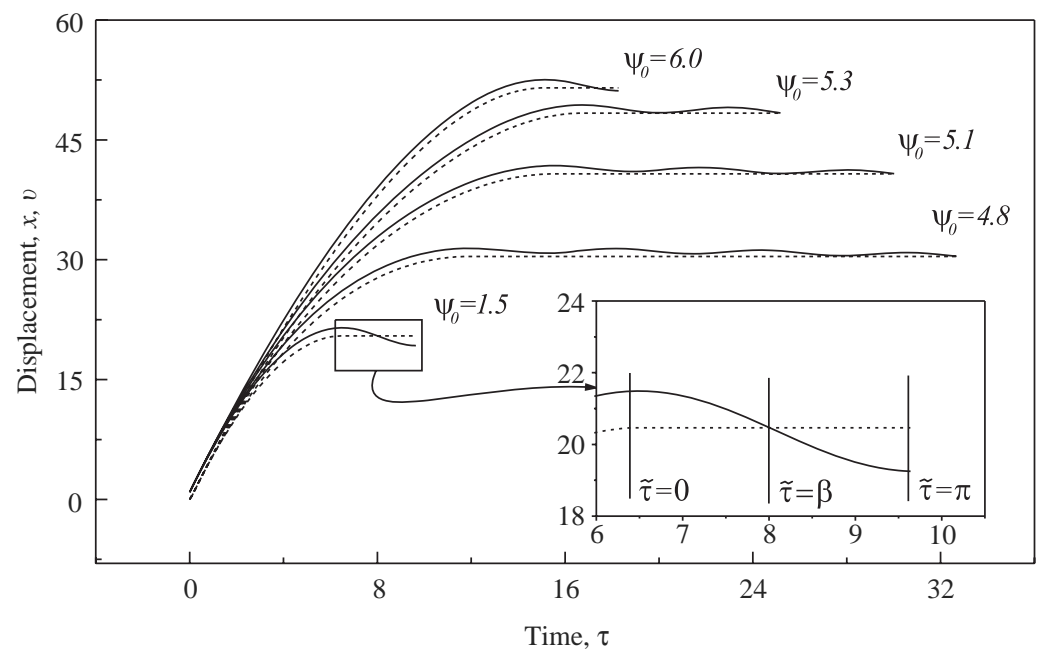

Fig. 3. Time histories of displacement of mass, $x$ (solid line) and displacement of slider bottom, $v$ (dashed line) calculated for $a=0.5, b=0.2, \xi=0.01, \omega=0.1, y_{0}=6$ and different initial phases $\psi_{0}$. 


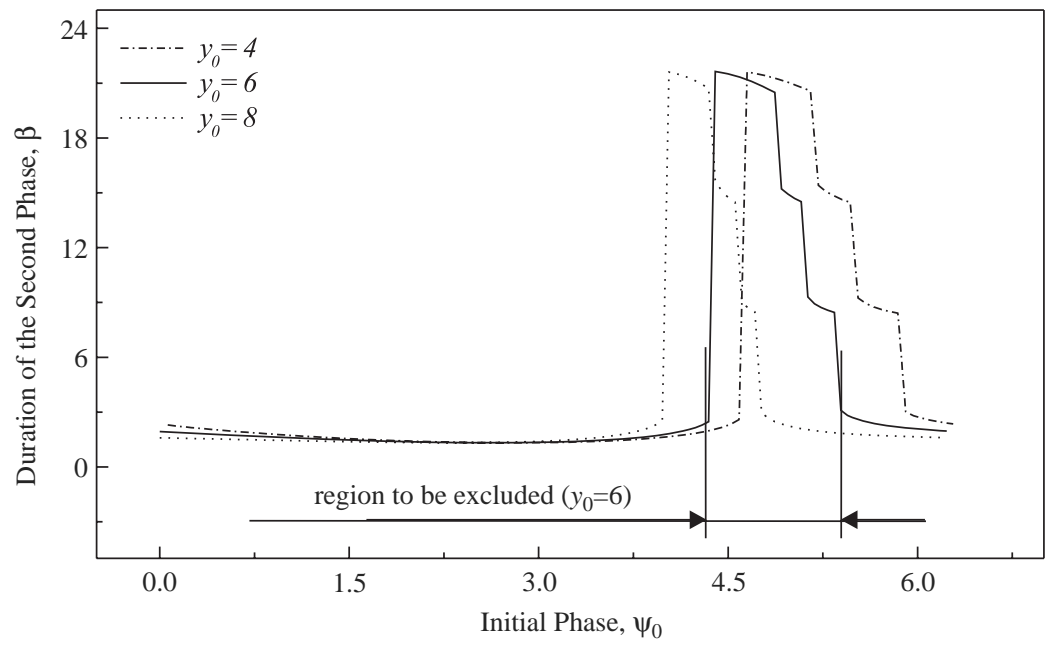

Fig. 4. Duration of the second phase $\beta$ as a function of $\psi_{0}$ at $a=0.5, b=0.2, \xi=0.01, \omega=0.1$ and different values of $y_{0}$.

the small zoom-up window that the system oscillates with the frequency equal to its natural frequency, $\omega_{n}=1$. This situation can occur for a range of $\psi_{0}$ prolonging the duration of the second phase. For example, if $\psi_{0}$ is chosen from the region $\psi_{0} \in(4.3,5.4)$ for $y_{0}=6$ as shown in Fig. 4 , the duration $\beta$ can be up to 10 times longer than one which is needed. This can be clearly seen from Fig. 4, where the desirable duration of the second phase equal to 2 is against 20, when the process settles down very slowly. Therefore, for $y_{0}=6$ the region of $\psi_{0}$ between 4.3 and 5.4 should be excluded. Practically, it means that only the values of the initial phase, $\psi_{0}$, where the duration $\beta$ is smaller than $\pi$ (a half of the period of oscillations when the mass is being in contact with the slider), should be considered. This can be achieved if the following condition is satisfied:

$$
\left.F_{1}\left(y_{0}, \psi_{0}\right) \equiv x\left(\tilde{\tau} ; y_{0}, \psi_{0}\right)\right|_{\tilde{\tau}=\pi}-\Delta\left(y_{0}, \psi_{0}\right)<0 .
$$

The first equation of (26) is used to determine function $x(\tilde{\tau})$.

\subsection{Phase III: no contact}

In the third phase, the mass and the slider top move separately, so integrating Eq. (4) one obtains

$$
\begin{gathered}
x(\tilde{\tau})=-\frac{a}{\omega^{2}}\left[\cos \left(\omega \tilde{\tau}+\psi_{2}\right)-\cos \left(\psi_{2}\right)\right]+\frac{1}{2} b \tilde{\tau}^{2}+y_{I I} \tilde{\tau}-\frac{a}{\omega} \tilde{\tau} \sin \left(\psi_{2}\right)+x_{I I}, \\
y(\tilde{\tau})=\frac{a}{\omega}\left[\sin \left(\omega \tilde{\tau}+\psi_{2}\right)-\sin \left(\psi_{2}\right)\right]+b \tilde{\tau}+y_{I I},
\end{gathered}
$$

where

$$
\tilde{\tau}=\tau-\alpha-\beta, \quad \psi_{2}=\psi_{0}+\omega(\alpha+\beta),
$$

and $x_{I I}, y_{I I}$ are defined by Eq. (29). 
Duration of the third phase, $\gamma$ can be found from the condition

$$
x(\gamma)=z(\gamma)+e,
$$

where $z(\gamma)=\left(z_{I I}-\Delta\right) \exp (-\gamma /(2 \xi))+\Delta$ is the solution of Eq. (5) at the moment $\tilde{\tau}=\gamma$. As the $\exp (-\gamma /(2 \xi))$ is very small, $x(\gamma) \approx \Delta+e$.

The condition (32) allows one to determine the moment when the mass hits the slider again (in this case, Eq. (31) is used to calculate $x(\tau)$ ). Again after substituting the expressions for $\alpha, \beta, \Delta, x_{I}$, $y_{I}, x_{I I}$ and $y_{I I}$ to Eq. (32), a non-linear algebraic equation for $\gamma$ is obtained:

$$
\gamma=\gamma\left(y_{0}, \psi_{0}\right) .
$$

As before, when $\gamma$ is found, the initial conditions for the next phase are calculated from Eq. (31):

$$
x_{I I I}=x(\gamma), \quad y_{I I I}=y(\gamma) .
$$

Durations $\gamma$ at chosen values of $y_{0}$ are depicted in Fig. 5 for different values of $\psi_{0}$ for $F_{1}<0$. As one is looking for the period equal to $2 \pi / \omega$, the duration $\gamma$ of the third phase needs to be relatively long. It means that the regions of $\psi_{0}$ where the trajectories of the mass and the slider bottom intersects only briefly, as those shown in Fig. 6, should be excluded. This can be achieved if one considers only the range of $\psi_{0}$, where the following condition is satisfied:

$$
\left.F_{2}\left(y_{0}, \psi_{0}\right) \equiv y\left(\tilde{\tau} ; y_{0}, \psi_{0}\right)\right|_{\tilde{\tau}=\tilde{\tau}_{*}}<0,
$$

the second equation of (31) is used to calculate $y(\tilde{\tau})$. The condition (35) means that in some moment $\tilde{\tau}_{*}$ of the third phase, the mass should still move in the opposite direction to the direction of progression. The value of $\tilde{\tau}_{*}$ should be appropriately chosen to avoid the region of $\psi_{0}$ where a jump of $\gamma$ occurs (see Fig. 5). Examining Fig. 5 one may deduce that the exclusion zone could start from the left dashed line, however, experience in solving the non-linear algebraic equation (32)

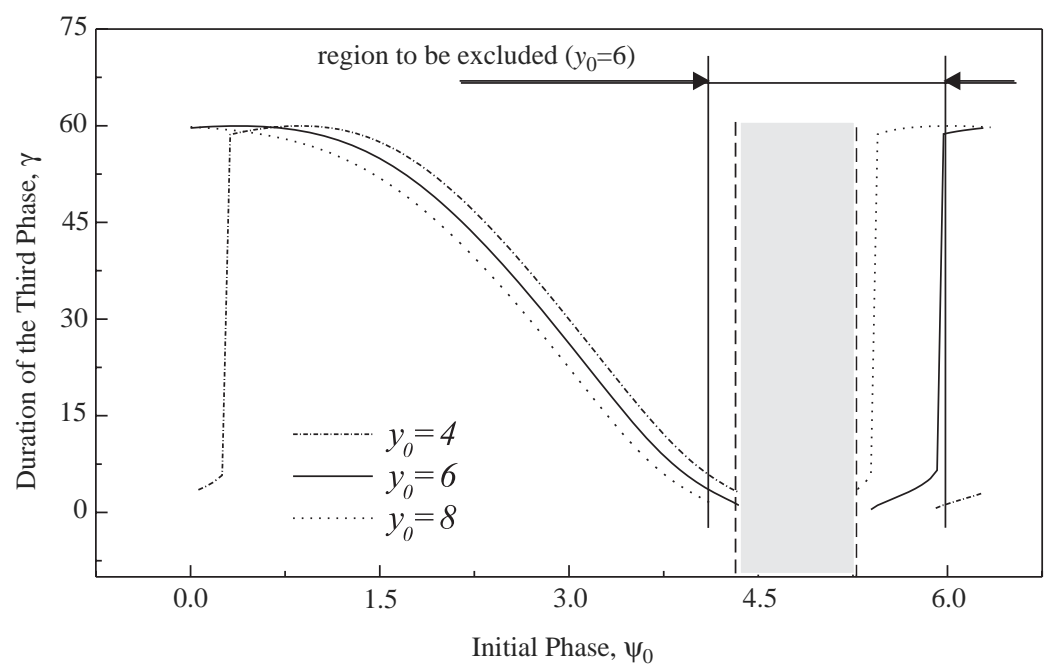

Fig. 5. Duration of the third phase $\gamma$ as function of $\psi_{0}$ for $a=0.5, b=0.2, \xi=0.01, \omega=0.1$ and different values of $y_{0}$. 


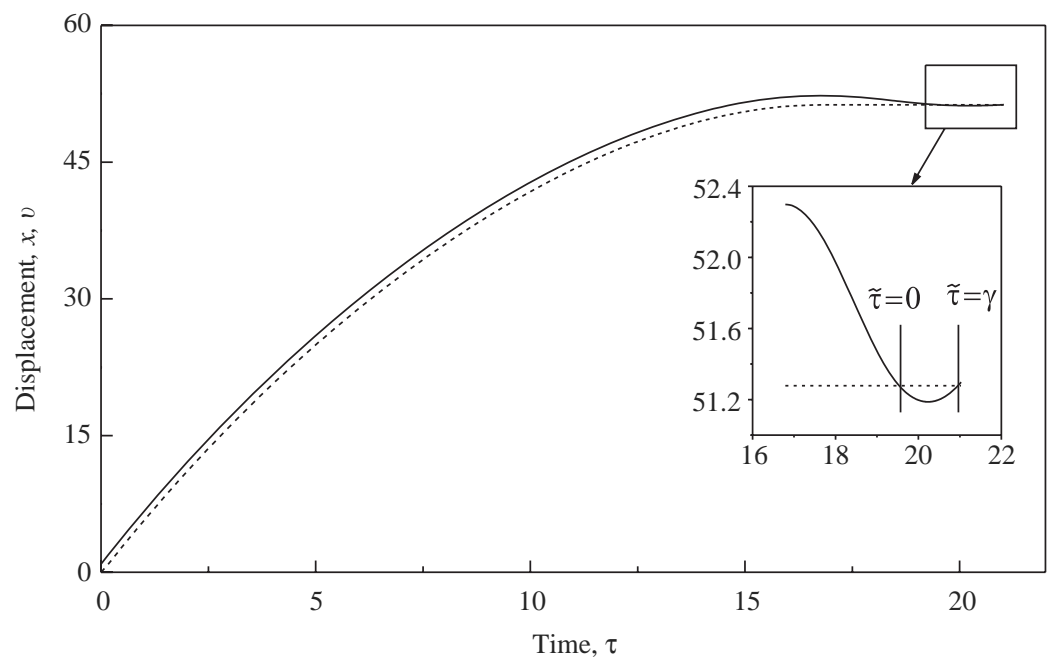

Fig. 6. Time histories of the mass (solid line) and slider bottom (dashed line) for $a=0.5, b=0.2, \xi=0.01, \omega=$ $0.1, y_{0}=6$, and $\psi_{0}=5.5$.

suggests, that it is more efficient to move this line to the left, in order to increase the numerical stability. A shaded area representing the exclusion zone from the second phase has been added to Fig. 5 to illustrate its location and extent. The final exclusion zone is a logical sum of these two exclusion zones.

\subsection{Phase IV: contact without progression}

In the last phase, $\delta$, the mass and the slider are moving together again up to the point, when the force acting on the slider is large enough to commence progression. Solution of Eq. (8) is given by Eq. (26) after introducing the following change of variables:

$$
\begin{gathered}
\tilde{\tau}=\tau-\alpha \rightarrow \tilde{\tau}=\tau-\alpha-\beta-\gamma, \\
\psi_{1}=\psi_{0}+\omega \alpha \rightarrow \psi_{3}=\psi_{0}+\omega(\alpha+\beta+\gamma), \\
x_{I} \rightarrow x_{I I I}, \\
y_{I} \rightarrow y_{I I I} .
\end{gathered}
$$

$\delta$ can be found from the following equation:

$$
2 \xi y(\delta)+x(\delta)-e-\Delta=1,
$$

where the functions $x$ and $y$ are calculated from Eqs. (26) and (27) using the substitution (36). The values of these functions for $\tilde{\tau}=\delta\left(x_{I V}\right.$ and $\left.y_{I V}\right)$ correspond to the displacement and velocity of the mass at the end of the period, $\tau=\alpha+\beta+\gamma+\delta$, and they should be substituted to the periodicity conditions (16). The periodic solutions with the period $T=2 \pi / \omega$ can only exist, if 
total duration of all four phases is equal to this period, so

$$
T=\alpha\left(y_{0}, \psi_{0}\right)+\beta\left(y_{0}, \psi_{0}\right)+\gamma\left(y_{0}, \psi_{0}\right)+\delta\left(y_{0}, \psi_{0}\right) .
$$

Thus, by using this condition the initial phase, $\psi_{0}$ can be represented as a function of the initial velocity, $y_{0}$ :

$$
\psi_{0}=\psi_{0}\left(y_{0}\right)
$$

In order to determine the initial velocity, $y_{0}$ for a chosen initial phase, $\psi_{0}$ one needs to find a minimum of the function $F\left(y_{0}\right)$ :

$$
F\left(y_{0}\right)=\sqrt{\left(x_{I V}\left(y_{0}\right)-\Delta\left(y_{0}\right)+2 \xi y_{0}-1-e\right)^{2}+\left(y_{I V}\left(y_{0}\right)-y_{0}\right)^{2}} .
$$

From $\partial F / \partial y_{0}=0$ first $y_{0}$ is calculated and then all unknown phases $\alpha, \beta, \gamma$ and $\delta$ are determined. If the minimum of the function $F\left(y_{0}\right)$ is equal to 0 , a periodic solution is found.

By employing the semi-analytical algorithm outlined in this section not only a period one solution can be determined but all periodic solutions, providing that the sequences of individual phases comprising different periods are defined a priori. The algorithm has proven to be robust in finding optimal parameters of the best progression for a given set of the system parameters and initial conditions. The latter are crucial as they can influence the progression rates significantly. In Fig. 7, a comparison between periodic and non-periodic solutions with respect to their time histories and progression per period is made. As can be seen a deviation from the optimal initial conditions has produced a significant decrease in progression per period.

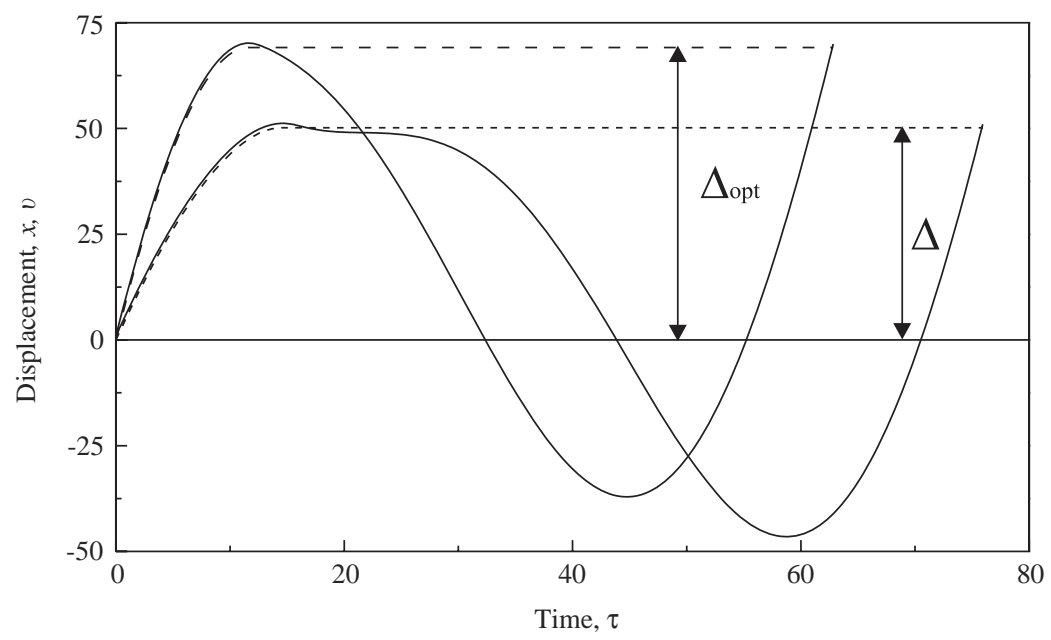

Fig. 7. Motion of the system during one period for $a=0.5, b=0.2, \xi=0.01$, and $\omega=0.1$. Displacement of mass, $x$, and the displacement of slider bottom, $v$ are marked by solid and dashed lines, respectively. An optimal periodic solution (thick lines) constructed for $y_{0}=10.88063, \psi_{0}=1.279532$ is confronted with a non-periodic one (thin lines) calculated for $y_{0}=6, \psi_{0}=6.1$. 


\section{Comparison with a direct numerical simulation}

The basic aim of the investigated system is to overcome the frictional force and move downwards. Despite of the fact that the considered model has only two degrees of freedom when the mass and the slider move separately, the dynamics of this system is very complex ranging from periodic to chaotic motion. In order to determine those various responses and assess the robustness of the devised semi-analytical solution, a direct numerical integration was used. Thus, the equations of motion were transformed to the following set of first order differential equations:

$$
\begin{gathered}
x^{\prime}=y, \\
y^{\prime}=a \cos (\omega \tau+\varphi)+b-P_{1} P_{2}\left(1-P_{3}\right)(2 \xi y+z-v)-P_{1} P_{3}, \\
z^{\prime}=P_{1} y-\left(1-P_{1}\right)(z-v) / 2 \xi, \\
v^{\prime}=P_{1} P_{3} P_{4}(y+(z-v-1) / 2 \xi),
\end{gathered}
$$

where a set of auxiliary functions, $P_{1}, P_{2}, P_{3}$ and $P_{4}$ of Heaviside type, is introduced. This allows one to describe more effectively the piecewise linear nature of the considered system

$$
\begin{gathered}
P_{1}=P_{1}(x, z)=\mathrm{H}(x-z-e), \\
P_{2}=P_{2}\left(z, z^{\prime}, v\right)=\mathrm{H}\left(2 \xi z^{\prime}+z-v\right), \\
P_{3}=P_{3}\left(z, z^{\prime}, v\right)=\mathrm{H}\left(2 \xi z^{\prime}+z-v-1\right), \\
P_{4}=P_{4}\left(v^{\prime}\right)=\mathrm{H}\left(v^{\prime}\right) .
\end{gathered}
$$

The numerical results shown in this section were computed using Dynamics software [21]. Eq. (41) was integrated using the fourth order Runge-Kutta algorithm with 1000 steps per one period of external excitation.

Non-linear dynamic analysis has been conducted in order to gain a fundamental insight and determine the optimal operational parameters. This paper restricts itself to only bifurcation diagrams, which have been constructed as follows. The initial value of the static force $(b)$ is set to the leftmost value in the figures. For this set of parameters, in order to exclude the transient behaviour, 100 cycles are calculated without plotting anything. The next 300 cycles brings 300 values of the velocity, $y$, which are plotted in the figure. Then a small increment is added to the branching parameter (in these figures it is equal to $\left.\left(b_{\max }-b_{\min }\right) / 480\right)$ and the procedure is repeated until the branching parameter reaches the rightmost value. A repetition of this procedure, by decreasing the branching parameter from rightmost to leftmost value, showed no hysteresis.

Typical bifurcation diagrams shown in Fig. 8 use the mass velocity to monitor the system dynamics due to the fact that the system moves forward. The other set of bifurcation diagrams depicted in Fig. 9 answers a practically important question 'How far will the system progress in a fixed time?' and use the bottom slider displacement. In the experiments, the time was set up to 50 

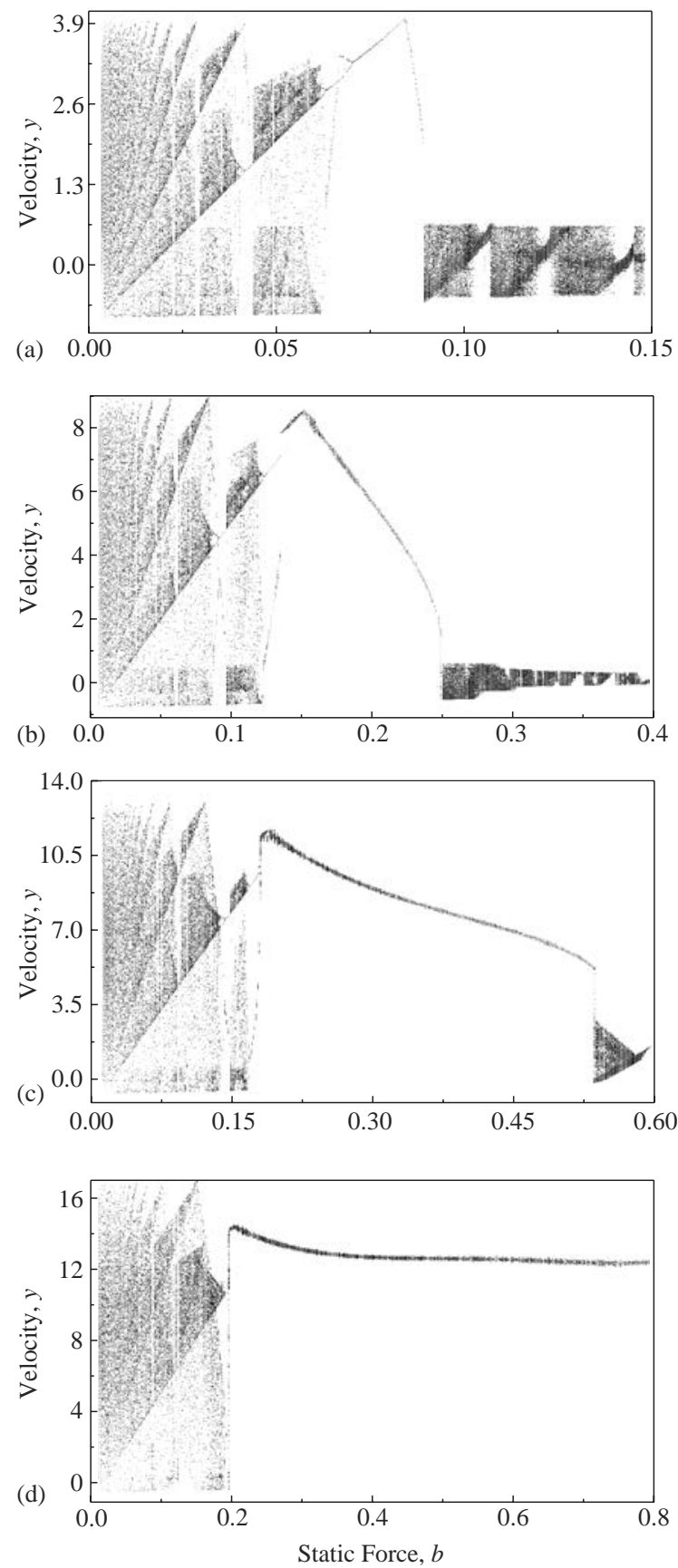

Fig. 8. Bifurcation diagrams $y=y$ (b) for $\xi=0.01, \omega=0.1$ and (a) $a=0.15$; (b) $a=0.4$; (c) $a=0.6$; (d) $a=0.8$. 

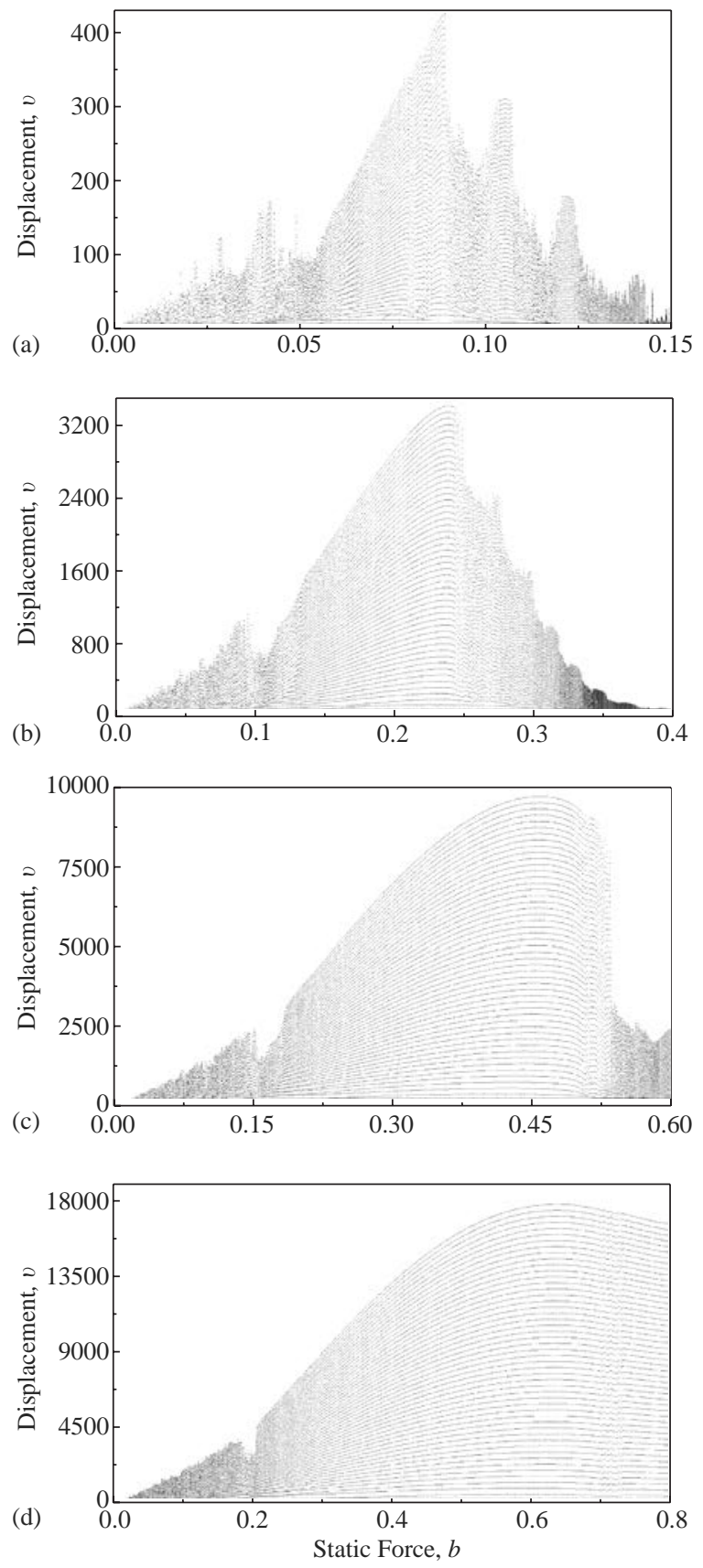

Fig. 9. Progression during the first 50 periods for $\omega=0.1, \xi=0.01$ : (a) $a=0.15$; (b) $a=0.4$; (c) $a=0.6$; (d) $a=0.8$. 


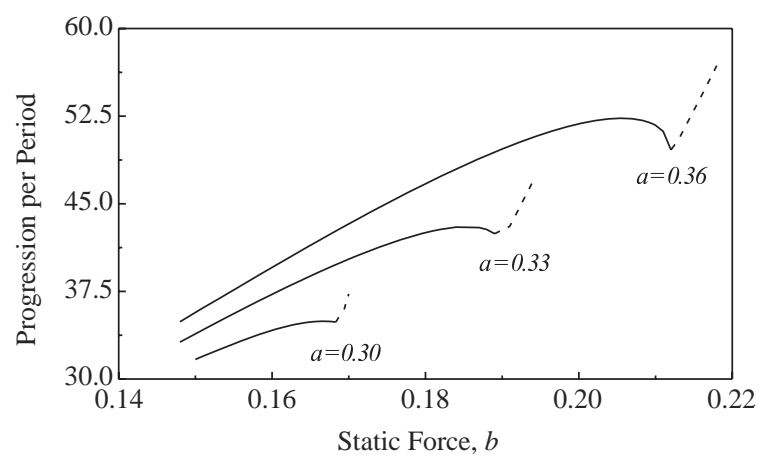

Fig. 10. Influence of static force, $b$ at $\xi=0.01, \omega=0.1$ calculated using the developed semi-analytical method.

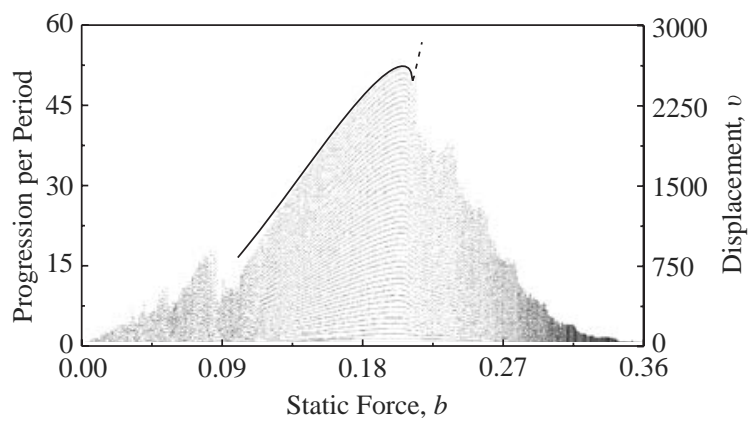

Fig. 11. Influence of static force, $b$ at $a=0.36, \xi=0.01, \omega=0.1$ computed numerically for 50 periods of excitation calculated semi-analytically (thick solid and dashed lines).

periods of external loading. As can be clearly seen from Fig. 9, the maximum penetration rate coincides with the end of periodic regime. It is also worth noting few local maxima for higher values of $b$.

The semi-analytical solution constructed in Section 3 allows one to study progression per period as a function of the system parameters. Fig. 10 shows the influence of the static force on the progression per period for different values of dynamic force under fixed damping coefficient and frequency of external loading. As it can be seen from the graphs, the progression curves have their maxima close to non-periodic solutions (dash lines). Although the dash lines show increases of the progression rates, the solutions obtained for these values of the static force are not periodic, so these predictions are false. It is worth noting that the existence of such maxima of progression is in good agreement with the experimental results [4] and the previous analytical models [7].

Fig. 11 shows a comparison between the semi-analytical and numerical predictions calculated for $a=0.36, \xi=0.1, \omega=0.1$, where the right vertical axis corresponds to the progression calculated numerically for the first 50 periods of external excitation. As can be seen from the figure, the results obtained from the developed semi-analytical method and the direct numerical integration are in a very good agreement. 

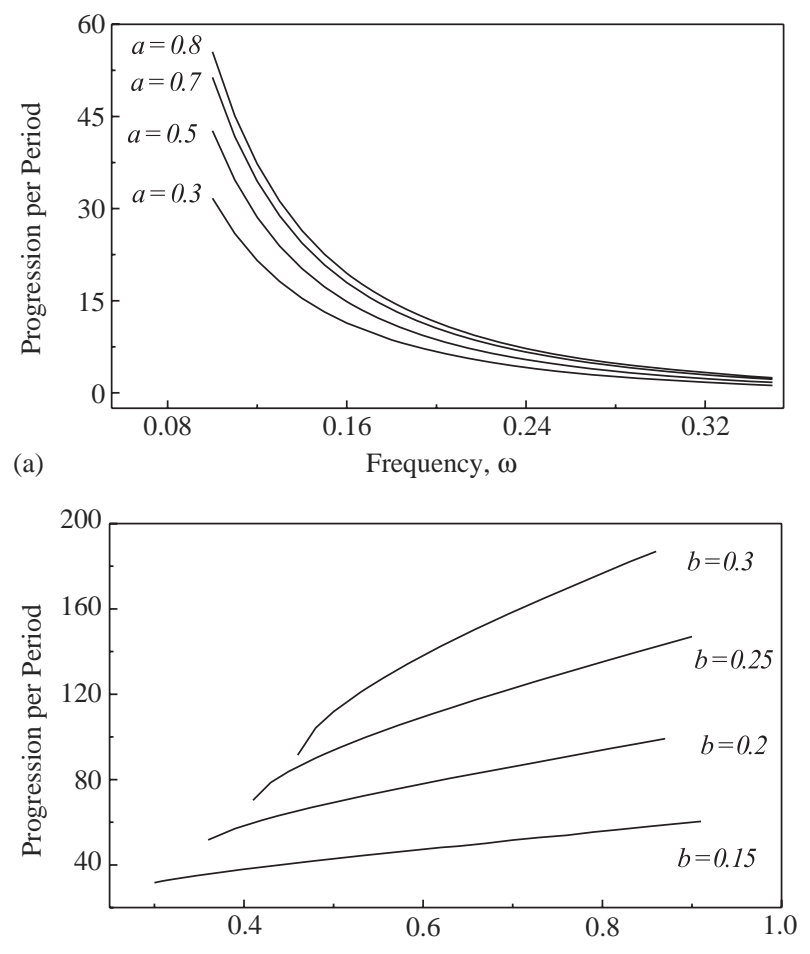

(b)

Dynamic Force, $a$

Fig. 12. Progression per period as a function of (a) frequency $\omega$ for $\xi=0.01, b=0.15$; (b) dynamic force $a$ for $\omega=0.1, \xi=0.01$.

The devised method has also been used to determine how the frequency and dynamic force influence the progression rates per period. Fig. 12a concludes that smaller external frequencies produce better progression rates. The monotonically decreasing curves indicate higher penetration rates for larger dynamic forces. The influence of the dynamic force on the progression per period is shown in Fig. 12b for different values of static force. The larger the dynamic and static force are, the larger the progressions per period are obtained.

\section{Closing remarks}

The paper gives a detailed account of an efficient semi-analytical method developed to calculate periodic responses for a new model of an impact system with a drift [5]. The method constructs a periodic solution assuming that each period is comprised of a sequence of distinct phases for which analytical solutions are known explicitly. For example, a period may consist of the following sequential phases: (I) contact with progression, (II) contact without progression, (III) no contact and (IV) contact without progression. Using this information, the system of four piecewise linear first order differential equations is transformed into a system of non-linear algebraic equations, which in turn are solved using a secant method due to its accuracy and speed. 
The algorithm is enhanced by two additional conditions (Eqs. (30) and (35)), which allow one to narrow down the search region. A comparison with direct numerical integration shows an excellent accuracy of the method. This algorithm can be used to predict a range of control parameters for which the best progression rates are achieved. Engineering applications of this method are foreseen in processes utilizing vibro-impact dynamics include percussive downhole drilling, ultrasonic drilling and ground moling.

\section{Acknowledgements}

The authors would like to acknowledge the financial support from EPSRC under the Grant GR/N16341/01.

\section{References}

[1] A. Benamar, Dynamic pile response using two pile-driving techniques, Soil Dynamics and Earthquake Engineering 20 (2000) 243-247.

[2] M. Wiercigroch, R.D. Neilson, M.A. Player, Material removal rate prediction for ultrasonic drilling of hard materials using impact oscillators approach, Physics Letters A 259 (2) (1999) 91-96.

[3] M. Wiercigroch, A. Krivtsov, J. Wojewoda, Dynamics of high frequency percussive drilling of hard materials, in: M. Wiercigroch, B. de Kraker (Eds.), Nonlinear Dynamics and Chaos of Mechanical Systems with Discontinuities, World Scientific, Singapore, 2000, pp. 403-444 (Chapter 16).

[4] K.-C. Woo, A.A. Rodger, R.D. Neilson, M. Wiercigroch, Application of the harmonic balance method to ground moling machines operating in periodic regimes, Chaos, Solitons and Fractals 11 (15) (2000) $2515-2525$.

[5] E. Pavlovskaia, M. Wiercigroch, C. Grebogi, Modelling of an impact system with a drift, Physical Review E 64 (2001) 056224.

[6] E.E. Pavlovskaia, M. Wiercigroch, K.-C. Woo, A.A. Rodger, Modelling of ground moling dynamics by an impact oscillator with a frictional slider, Meccanica 38 (1) (2003) 85-97.

[7] A.M. Krivtsov, M. Wiercigroch, Dry friction model of percussive drilling, Meccanica 34 (6) (1999) $425-434$.

[8] A.M. Krivtsov, M. Wiercigroch, Penetration rate prediction for percussive drilling via dry friction model, Chaos, Solitons and Fractals 11 (15) (2000) 2479-2485.

[9] P.J. Holmes, Dynamics of repeated impacts with a sinusoidally vibrating table, Journal of Sound and Vibration 84 (2) (1982) 173-189.

[10] J.M.T. Thompson, R. Ghaffari, Chaotic dynamics of an impact oscillator, Physical Review A 27 (1983) 1741-1743.

[11] F.C. Moon, S.W. Shaw, Chaotic vibrations of a beam with non-linear boundary conditions, International Journal of Nonlinear Mechanics 18 (6) (1983) 465-477.

[12] A.B. Nordmark, Non-periodic motion caused by grazing incidence in an impact oscillator, Journal of Sound and Vibration 145 (2) (1991) 279-297.

[13] S. Foale, S.R. Bishop, Dynamic complexities of forced impacting systems, Philosophical Transactions of the Royal Society of London A 338 (1992) 547-556.

[14] C. Budd, F. Dux, Chattering and related behaviour in impact oscillators, Philosophical Transactions of the Royal Society of London A 347 (1994) 365-389.

[15] V.T.W. Sin, M. Wiercigroch, Symmetrically piecewise linear oscillator: design and measurement, Proceedings of the Institution of Mechanical Engineers C 213 (1999) 241-249.

[16] M. Wiercigroch, V.T.W. Sin, Experimental study of a symmetrical piecewise base-excited oscillator, American Society of Mechanical Engineers, Journal of Applied Mechanics 65 (1998) 657-663. 
[17] A.E. Kobrinskii, Dynamics of Mechanisms with Elastic Connections and Impact Systems, ILIFFE Books Ltd., London, 1969.

[18] V.I. Babitsky, Hand-held percussion machine as discrete non-linear converter, Journal of Sound and Vibration 214 (1) (1998) 165-182.

[19] M. Wiercigroch, A note on the switch function for the stick-slip phenomenon, Journal of Sound and Vibration 175 (5) (1994) 700-704.

[20] U. Galvanetto, S.R. Bishop, Stick-slip vibrations of a 2-degree-of-freedom geophysical fault model, International Journal of Mechanical Sciences 36 (8) (1994) 683-698.

[21] H.E. Nusse, J.A. Yorke, Dynamics: Numerical Explorations, Springer, New York, 1998. 